\title{
An emerging potential therapeutic target for osteoporosis: IncRNA H19/miR-29a-3p axis
}

\author{
Ziqi Li, ${ }^{1}$ Zhinan Hong, ${ }^{1}$ Yuesheng Zheng, ${ }^{2}$ Yongwei Dong, ${ }^{2}$ Wei He, ${ }^{2}$ Yingjia Yuan, ${ }^{1}$ Junbiao Guo ${ }^{3}$ \\ ${ }^{1}$ Department of Joint Disease, Traumatology and Orthopedics institute of Guangzhou University of Chinese Medicine, \\ the third affiliated hospital of Guangzhou University of Chinese Medicine, Guangzhou \\ ${ }^{2}$ Department of Orthopedics, Jinshazhou hospital of Guangzhou University of Chinese Medicine, Guangzhou \\ ${ }^{3}$ Guangdong Provincial Hospital of Chinese Medicine, The Second Affiliated Hospital of Guangzhou University of \\ Chinese Medicine, Guangzhou, China
}

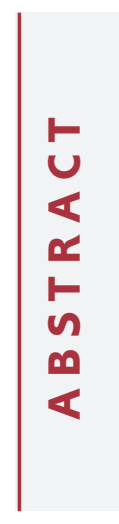

\begin{abstract}
Osteoporosis (OP) is a complex systemic disease characterized by a loss of bone density, leading to bone fragility and an increased risk of fractures of the hip, spine and wrist. The clinical therapeutic effect is still far from satisfactory. Thus, further studies are urgently needed to explore the pathogenesis of OP. In this study, our aim was to explore the underlying molecular mechanism of lncRNA H19/miR-29a-3p axis for regulating of inflammation, proliferation and apoptosis in OP. The expression of lncRNA H19 was significantly upregulated in OP samples compared with the health control. Subsequently, we found that miR-29a-3p is the target of IncRNA H19 in OP. Furthermore, the knockdown of lncRNA H19 was validated to promote the expression of pro-inflammatory mediators, repress cell proliferation and inhibit cell apoptosis in vitro. Moreover, the modulating effects of IncRNA-H19 on the expressions of pro-inflammatory mediators, cell proliferation and apoptosis in vitro were diminished after co-transfecting with miR-29a-3p inhibitor and siRNA-H19. Thus, we concluded that lncRNA H19/miR-29a-3p axis was involved in the development of OP. This study might provide a better understanding of OP development and a potential therapeutic target for OP intervention.
\end{abstract}

Key words: Target therapy; lncRNA H19; miR-29a-3p; osteoporosis.

Correspondence: Junbiao Guo, PhD, Guangdong Provincial Hospital of Chinese Medicine, the second affiliated hospital of Guangzhou University of Chinese Medicine, Guangzhou, Guangdong, China.

E-mail: 599212513@qq.com

Contributions: ZL and ZH contribute equally to this study. YY, JG, conceived the study and designed the experiments; ZL, ZH, performed the experiments and wrote up the manuscript; YZ, YD, WH, contributed to the sample collection and data analysis. All authors read and approved the final version and agreed to be accountable for all aspects of the work.

Conflict of interest: The authors declare that they have no competing interests, and all authors confirm accuracy.

Ethics approval and consent to participate: Ethical approval was obtained for all experimental procedures by the Ethics Committee of the Guangzhou University of Chinese Medicine, Guangzhou, Guangdong, China. All participants signed informed consents before the study.

Availability of data and materials: The data used to support the findings of this study are available from the corresponding author upon request. 


\section{Introduction}

Osteoporosis (OP) is a prevalent metabolic bone disease with the bone fragility. OP affects millions of individuals worldwide, particularly those with pathologic fractures. ${ }^{1,2}$ OP has been identified as one of the three major chronic diseases with hypertension and diabetes, which is mostly related to estrogen and age. Currently, hormone replacement, calcium supplement and inhibition on bone resorption are main clinical therapeutic methods for OP. However, the clinical therapeutic effect is still far from satisfactory since the toxic and side effects for drugs, malabsorption and poor patient compliance. Further researches are therefore urgently needed to explore the potential therapeutic targets of OP. ${ }^{3}$ Long non-coding RNAs (lncRNAs) are transcribed from the nonprotein part of human genome with more than 200 nucleotide (nt) in length. lncRNAs are widely involved in the regulation of gene expression in various kinds of diseases. ${ }^{4-7}$

Several studies have found that lncRNAs play important roles in the occurrence and development of OP. IncRNA CASC11 has been found to be upregulated in postmenopausal OP and is correlated with TNF- $\alpha .{ }^{8}$ In addition, lncRNA TUG1 was upregulated in OP and regulates the proliferation and apoptosis of osteoclasts. ${ }^{9}$ lncRNA H19 has been reported in several studies to be involved in the development and progression of various types of diseases. lncRNA H19 rs217727 polymorphism has been found to be associated with oral squamous cell carcinoma susceptibility in Iranian population. ${ }^{10}$ IncRNA H19 has also been found to promote tumorigenesis of multiple myeloma by activating BRD4 signaling by targeting miR-152-3p. ${ }^{11}$ Moreover, IncRNA H19 has been validated as a novel therapeutic target for pancreatic cancer. ${ }^{12}$ However, the functional roles of lncRNA H19 in the progression and development of OP still remain largely unclear.

MicroRNAs (miRNAs) are endogenous short non-coding RNAs with 17-25 nt in length that control gene expression at the post-translational level by mRNA degradation and translational repression. ${ }^{13}$ In recent years, researchers have explored the roles of lncRNA/miRNA loop in the progression and development in many kinds of diseases. IncRNA MSC-AS1 promoted osteogenic differentiation and alleviates OP through sponging miRNA-140-5p to upregulate BMP2. ${ }^{14}$ In addition, miR-29a-3p has been reported as the target of lncRNA H19 in the cell renal cell carcinoma. ${ }^{15}$ Moreover, lncRNA MALAT1 shuttled by bone marrow-derived mesenchymal stem cells-secreted exosomes alleviates OP through mediating miRNA-34C/SATB2 axis. ${ }^{16}$ However, lncRNAH19/miR-29a-3p whether functions as regulatory mediator has not been reported in OP.

In the present study, our goal is to elucidate the underlying molecular mechanism of lncRNA H19/miR-29a-3p axis for regulating of inflammation and proliferation in OP. The differentially expressed lncRNA H19, and the expression of miR-29a-3p in in
OP and health control samples were firstly determined by qRTPCR. Subsequently, we detected the underlying mechanism of lncRNA-H19/miR-29a-3p in the development of OP in vitro. This study might provide a novel understanding of OP development and a potential window for OP therapeutic intervention.

\section{Materials and Methods}

\section{Sample collection}

In this study, verbal consent was obtained from all subjects before sample collection. A total of 30 female subjects were recruited into present study, including 15 patients at diagnosis with postmenopausal OP (age: 45-75 years) and 15 postmenopausal controls (age: 43-76 years) from Guangdong Provincial Hospital of Chinese Medicine between February 2019 and November 2019. The inclusion criteria were as follows: natural menopause after 40 years of age and a bone mineral density (BMD) of at least 2.5 standard deviation (SD) below the peak mean BMD of healthy young women (-2.5 T-score) at the lumbar spine, total hip or femoral neck. Patients with a medical history of OP treatment, hormone replacement therapy, early menopause ( $<40$ years), abnormal menopause, acute gastrointestinal inflammation, or chronic renal failure were excluded. Bone marrow samples were collected during the implantation of a total endoprosthesis or gamma nail into the proximal femur. Five $\mathrm{mL}$ of venous blood was harvested from each clinically diagnosed OP patients and controls in the morning under the fasting stage. Their pathological data were then collected. Blood samples were centrifuged $(3000 \mathrm{~g} / \mathrm{min}, 10 \mathrm{~min})$ at $4{ }^{\circ} \mathrm{C}$ after standing for $30 \mathrm{~min}$. The supernatant serum (non-hemolytic state) was centrifuged $(135,000 \mathrm{~g} / \mathrm{min}, 15 \mathrm{~min})$ again at $4^{\circ} \mathrm{C}$. After then, the serum samples were sub-packaged $(200 \mu \mathrm{L} /$ tube $)$ and stored at $-80^{\circ} \mathrm{C}$ for subsequent use. All protocols were approved by the Ethics Committee of Guangzhou University of Chinese Medicine, and all participants signed informed consent before the study.

\section{qRT-PCR}

We used RNAzol reagent (Sigma-Aldrich, Shanghai, China) to extract total RNA from plasma. The quantity of RNA was measured using the QIAxpert (Qiagen, Shenzhen, China). After removal of residual DNA by TURBO DNase (Thermo Fisher Scientific, Shanghai, China), High-Capacity cDNA Reverse Transcription Kit (Thermo Fisher Scientific) was used to performed reverse transcription. To detect the expression of lncRNA H19, TNF- $\alpha$, IL-1 $\beta$ and IL-10 mRNAs, primers including $\beta$-actin were synthesized by IDT (San Diego, CA, USA). $\beta$-actin was used as endogenous reference for normalization. All primers are listed in Table 1.

Total RNA was reverse-transcribed using the Taqman

Table 1. All primers for qRT-PCR.

\begin{tabular}{lcc} 
& Forward primer & Reverse primer \\
IncRNA H19 & ATCGGTGCCTCAGCGTTCGG & TGTCCTCGCCGTCACACCG \\
TNF- $\alpha$ & GCCTCTTCTCATTCCTGCTTG & CTGATGAGAGGGAGGCCATT \\
\hline IL-1 $\beta$ & TGGACCTTCCAGGATGAGGACA & GTTCATCTCGGAGCCTGTAGTG \\
IL-10 & CGGGAAGACAATAACTGCACCC & CGGTTAGCAGTATGTTGTCCAGC \\
\hline$\beta$-actin & CGTGCGTGACATCAAAGAGAA & TGGATGCCACAGGATTCCAT \\
\hline
\end{tabular}


advanced miRNA cDNA synthesis kit (Applied Biosystems, Foster City, CA, USA) to determine the expression of miR-29a-3p. The expression of miR-29a-3p was normalized to U6 small nuclear RNA (snRNA). U6 was reverse transcribed by Taqman ${ }^{\mathrm{TM}}$ microRNA reverse transcription kit (Applied Biosystems). miR29a-3p Taqman probe is commercially available from Applied Biosystems. All of the relative expression levels were calculated in according with the $2^{-\Delta \Delta \mathrm{CT}}$ method. ${ }^{17}$.

\section{Osteoclasts}

Primary marrow-derived osteoclasts were generated from bone marrow osteoclast precursors. All operations were strictly followed the previously described methods. ${ }^{18}$ Briefly, to obtain primary osteoclasts, bone marrow was flushed from long bones of 4to 6-week-old C57BL/6 mice (Guangdong Medical Experimental Animal Center, Guangdong, China). Cells were cultured in $\alpha$ MEM (Gibco-Invitrogen, Carlsbad, CA, USA) supplemented with 10\% FBS (Gibco-Invitrogen), 2 mM L-glutamine (Sigma-Aldrich, Shanghai, China), and $1 \% \mathrm{P} / \mathrm{S}$ (Sigma-Aldrich) for $2 \mathrm{~d}$ before collecting the nonadherent, hematopoietic bone marrow cells for experiments.

\section{Luciferase reporter assay}

Dual-luciferase assay was performed to confirm the interaction between lncRNA-H19 and miR-29a-3p. IncRNA H19 wild-type luciferase reporter vector (H19-WT) containing miR-29a-3p targeted sequence and its mutant in the seeded region (H19-MUT) were constructed by Applied Biosystems. Osteoclasts were transfected with the reporter plasmids and miR-29a-3p or miRNA control (miR-con) with lipofectamine 2000 (Thermo Fisher Scientific) as previously described. ${ }^{19}$ Data was expressed as a ratio of firefly luciferase activity normalized to Renilla luciferase activity.

\section{RNA immunoprecipitation assay}

We determined the interaction between H19 and miR-29b-3p using immunoprecipitation (RIP) assay as previously described. ${ }^{15}$ EZMagna RIP RNA-binding protein immunoprecipitation kit (Millipore, Billerica, MA, USA) was carried out according to the manufacturer's instructions. RNA was pulled down with the antihuman argonaute2 (Ago2) antibodies (Millipore), and IgG (Millipore) was used as a negative control (input). The coprecipitated RNAs were reverse-transcribed and analyzed by qRT-PCR to detect the enrichment of lncRNA-H19 and miR-29a-3p.

\section{Overexpression and knockdown}

pcDNA3.1-H19, pcDNA3.1, H19 targeting siRNA (si-H19) and its negative control (si-NC) were purchased from Genepharma Inc. (Shanghai, China). Osteoclasts transfection with pcDNAs or siRNAs were carried out by Lipofectamine 2000 (Thermo Fisher Scientific) according to the manufacture protocol. The miR-29a-3p inhibitor and negative control was bought from Genepharma.

\section{Cell proliferation assay}

We used cell counting kit-8 (CCK-8; Dojindo Laboratories, Kumamoto, Japan) to detect the proliferation of cells. The cells (2 $\times 10^{4}$ cells $/ \mathrm{mL}$ ) were seeded in a 96-well plate. Subsequently, the CCK-8 solution $(10 \mu \mathrm{L} /$ well $)$ was added at $12,24,48$, or $72 \mathrm{~h}$ to monitor cell viability. After then, the cells were incubated with $5 \%$ $\mathrm{CO}_{2}$ at $37^{\circ} \mathrm{C}$ for $2 \mathrm{~h}$. The absorbance of each well was measured at $450 \mathrm{~nm}$ using a microplate reader.

\section{Flow cytometric analysis}

Cells $\left(5 \times 10^{5}\right.$ cells /well) with transfection of si-NC, si-H19, miR-29-3p inhibitor, or si-H19 + miR-29-3p were cultured in the 6-well plates for $48 \mathrm{~h}$. Cells were harvested and apoptotic cells were detected by staining with Annexin V-FITC and propidium iodide (PI) according to the manufacturer's protocol (BD Biosciences, Shanghai, China). The stained cells were immediately analyzed by a flow cytometer.

\section{Statistical analysis}

Statistical analyses were performed by using the SPSS 22.0 software (SPSS Inc, Chicago, IL, USA). All data were expressed as mean $\pm \mathrm{SE}$ in 3 independent experiments with triplicate measurements. We used Student's paired $t$-test to analyze the differences between groups. Significant differences were considered when a p-value less than 0.05 .

\section{Results}

\section{Expression of IncRNA H19 in plasma}

The plasma of patients with OP $(n=15)$ and controls $(n=15)$ were used to determine the expression of lncRNA H19 using qRTqPCR. IncRNA H19 expression were significantly higher in the plasma of OP patients relative to healthy participants (Figure 1A, $\mathrm{P}<0.05)$.

\section{MiR-29a-3p is the target of IncRNA H19}

We subsequently exanimated the expression level changes of miR-29a-3p in the plasma between OP and healthy participants using qRT-PCR. As shown in Figure 2A, the expression of miR$29 a-3 p$ was remarkedly decreased in the plasma of OP participants than that of healthy participants.

To explore whether there is a direct effect between lncRNA

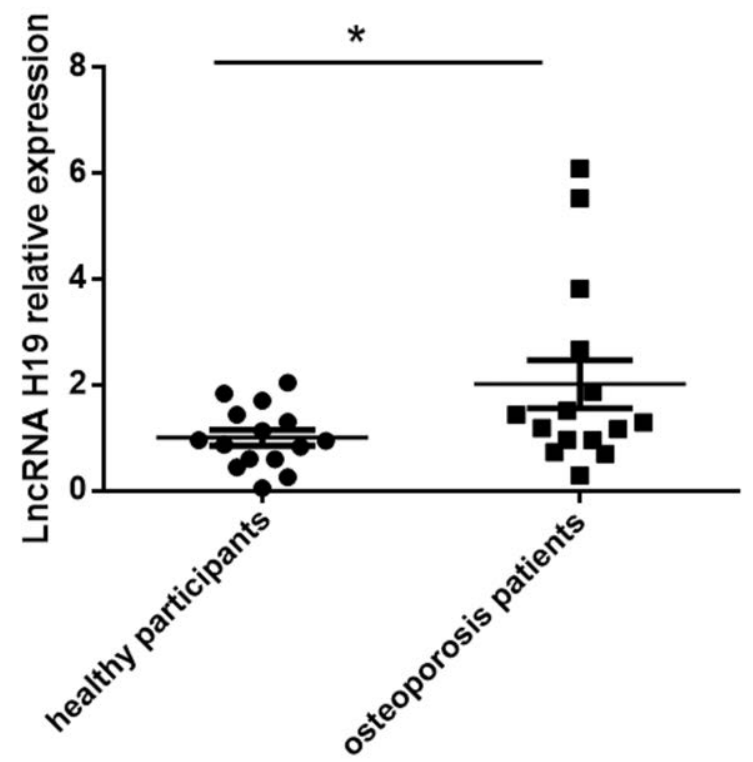

Figure 1. IncRNA H19 was highly expressed in the plasma of OP patients than in healthy participants. The $\mathrm{QRT}-\mathrm{PCR}$ results showed that plasma levels of IncRNA H19 were significantly upregulated in OP patients compared with healthy participants. ${ }^{*} \mathrm{p}<0.05$ vs health participates. 
H19 and miR-29a-3p, dual-luciferase assay was carried out in osteoclasts. We found that miR-29a-3p suppressed the luciferase activity of H19-WT but not H19-MUT (Figure 2 B,C). To confirm that lncRNA H19 associates with miR-29a-3p, RIP was performed after then to detect the binding abundances of lncRNA H19, miR$29 a-3 p$ and Ago 2 . The results illustrated that the expression abun- dance of lncRNA H19 and miR-29a-3p in the Ago2 antigen-antibody complex were significantly increased compared with the control group (Figure 2D). This indicated that lncRNA H19 and miR29a-3p can be combined with Ago2, which was consistent with the luciferase assay.
A
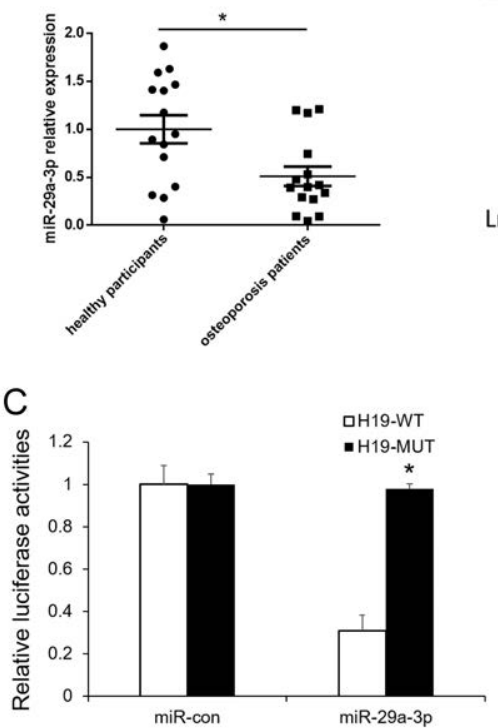

B
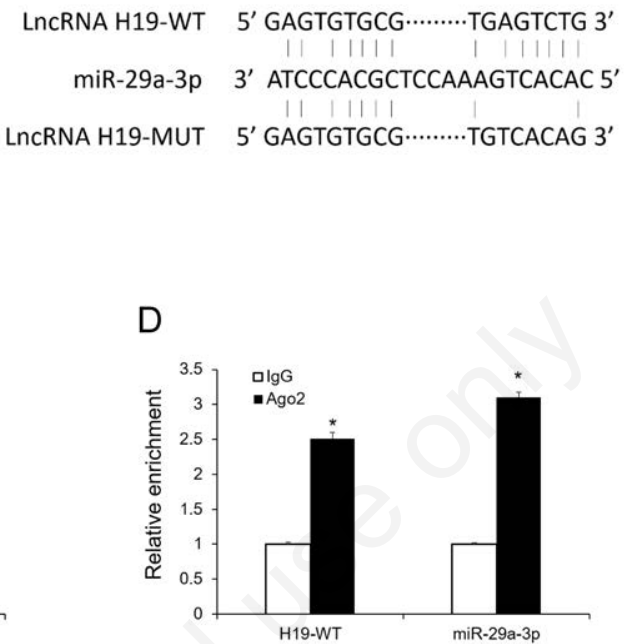

Figure 2. MiR-29a-3p is the target of IncRNA H19. A) miR-29a-3p was decreased in the plasma of OP patients than in healthy participants; qRT-PCR results showed that plasma miR-29a-3p levels were significantly upregulated in OP patients compared with healthy participants; ${ }^{*}$ p $<0.05$ vs health participates. B) Representation the wildtype lncRNA-H19 (WT) and mutant (MUT) with the miR-29a3 p binding sites. C) Confirm the interaction between miR-29a-3p and lncRNA H19 by dual-luciferase assay; sata were represented as the mean \pm SE $(n=3),{ }^{*} p<0.05$ vs H19-WT. D) lncRNA H19 mRNA level was detected in the substrate of RIP assay by $q R T-P C R ;$ data were represented as the mean $\pm \mathrm{SE}(\mathrm{n}=3),{ }^{*} \mathrm{p}<0.05$ vs $\mathrm{IgG}$.
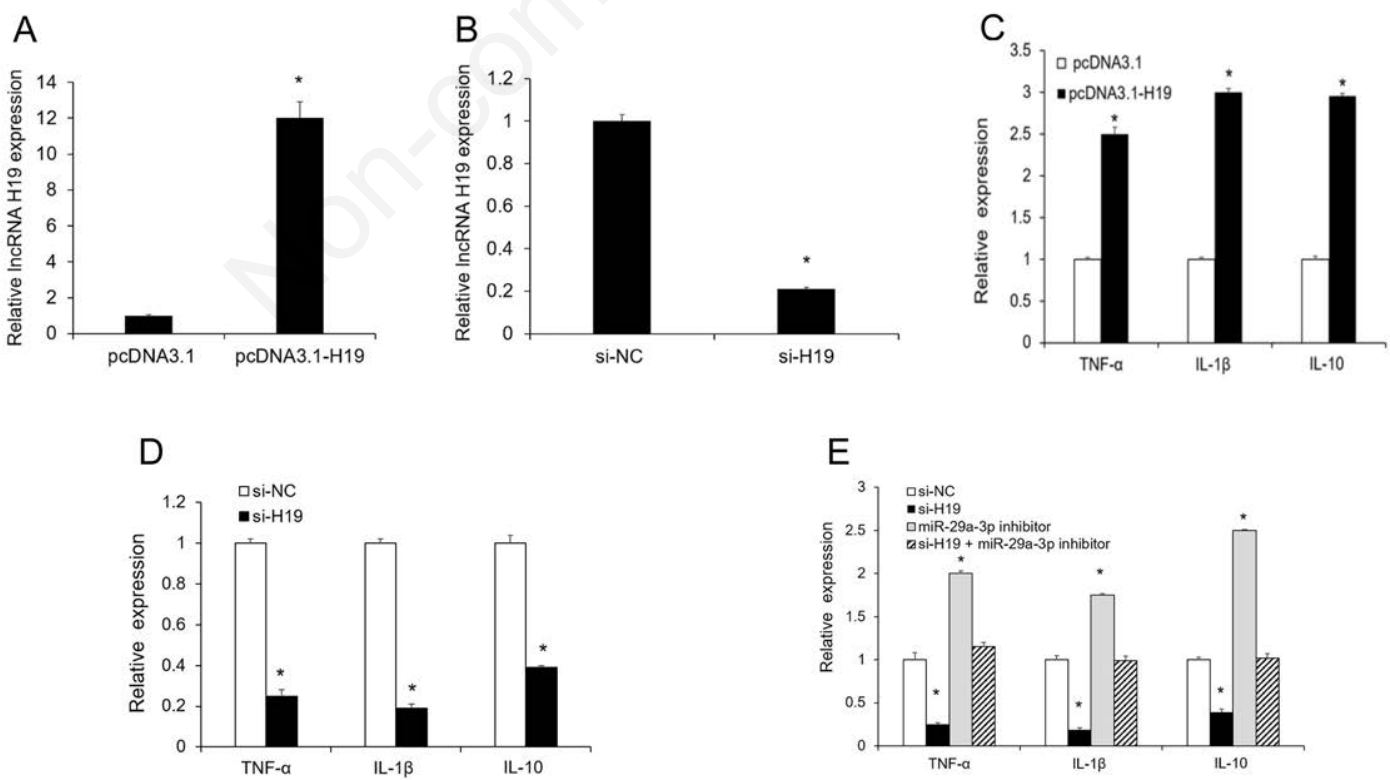

Figure 3. IncRNA-H19 regulated the expression of pro-inflammatory factors through miR-29a-3p. A) The mRNA level of lncRNA H19 in cells transfected with pcDNA3.1 or pcDNA3.1-H19. B) The mRNA level of IncRNA H19 in cells transfected with si-NC or si-H19. C) The mRNA level of TNF- $\alpha$, IL-1 $\beta$ and IL-10 in cells transfected with pcDNA3.1 or pcDNA3.1-H19. D) The mRNA level of TNF$\alpha$, IL-1 $\beta$ and IL-10 in cells transfected with si-NC or si-H19. E) The mRNA level of TNF- $\alpha$, IL-1 $\beta$ and IL-10 in cells transfected with si-NC, si-H19, miR-29-3p inhibitor, or si-H19 + miR-29-3p inhibitor. All data were represented as the mean $\pm \mathrm{SE}(\mathrm{n}=3)$, ${ }^{*} \mathrm{p}<0.05$ vs control. 
IncRNA H19/miR-29a-3p promoted the expression of pro-inflammatory factors

Overexpression of IncRNA H19 has been reported to be relative to the inflammation response. ${ }^{20}$ We initially explored the effects of IncRNA H19/ miR-29a-3p axis on the inflammation response of OP. We firstly confirmed that lncRNA H19 was successfully overexpressed and silenced in osteoclasts. As present in Figure $3 \mathrm{~A}, \mathrm{~B}$, lncRNA H19 expression was significantly increased and decreased after transfecting with pcDNA3.1-H19 or si-H19 compared with their related controls. IncRNA H19 overexpression promoted the expression of in osteoclasts (Figure 3C). However, the knockdown of IncRNA H19 suppressed the expressions of TNF- $\alpha$, IL-1 $\beta$ and IL-10 in osteoclasts (Figure 3D). Moreover, the modulating effects of IncRNA-H19 on the expressions of TNF- $\alpha$, IL-1 $\beta$ and IL-10 were diminished in the cells of co-transfecting with miR-29a-3p inhibitor and siRNA-H19 (Figure 3E).

\section{IncRNA H19/miR-29a-3p axis on cell proliferation}

IncRNA H19 has been reported to have modulating effect on cell proliferation, ${ }^{15}$ it is unclear whether lncRNA H19 has effects on proliferation of osteoclasts. We subsequently explored the effects of lncRNA H19/miR-29a-3p on cell proliferation. As we expected, osteoclasts cell proliferation was significantly repressed at the different indicated time points using a CCK- 8 assay after cells were transfected with si-H19 (Figure 4). However, co-transfecting with miR-29a-3p inhibitor and siRNA-H19 reduced the modulating effects of siRNA-H19 on the cell proliferation (Figure 4).

\section{IncRNA H19 modulated the cell apoptosis via miR-29a-3p}

The effect of IncRNA H19/miR-29a-3p on the cell apoptosis was determined by cell flow cytometry. Osteoclasts cell apoptosis was significantly inhibited after cells were transfected with si-H19 (Figure 5). However, co-transfecting with miR-29a-3p inhibitor and siRNA-H19 obligated the effects of siRNA-H19 on the cell proliferation (Figure 5).

To sum up, IncRNA H19/miR-29a-3p axis contributes to the development of OP via modulating the expression of pro-inflammatory factors, proliferation and apoptosis. Our findings at least partly explain the underlying molecular mechanism for the regulatory effect of lncRNA H19/miR-29a-3p axis.

\section{Discussion}

OP is a commonly occurring condition marked by destruction of balance between bone formation of osteoblasts and bone resorption of osteoclasts because of estrogen deficiency. Since OP patients have a limited option of clinical therapeutic option, thus the explore of novel molecular markers are required. However, the function of lncRNA H19 has not been elucidated in OP. In this study, we first found lncRNA H19 was significantly upregulated in OP plasma than that of health control samples. Furthermore, miR$29 a-3 p$ has been found as the target of lncRNA H19. Moreover, lncRNA H19 has been verified to elevate the expression of proinflammatory mediators, such as TNF- $\alpha$, IL-1 $\beta$ and IL-10, and enhance the proliferation.

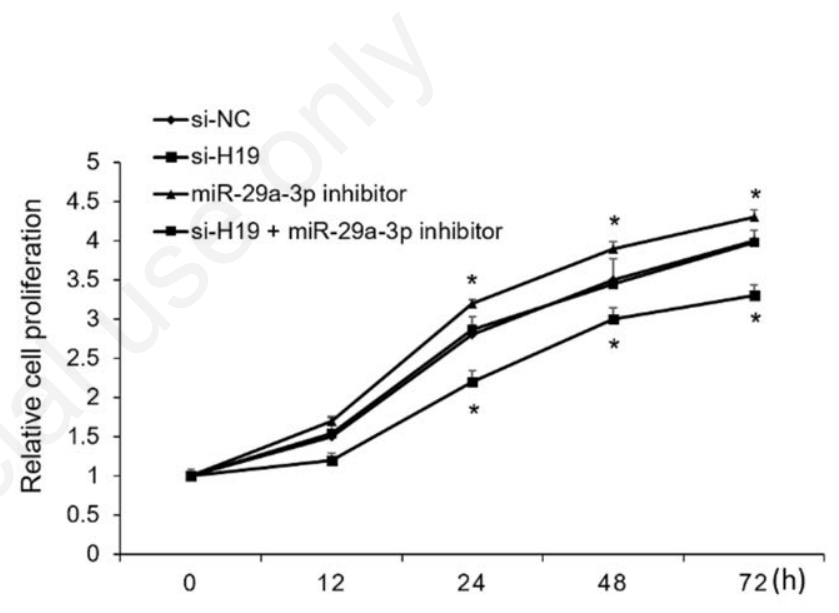

Figure 4. IncRNA H19 modulated the cell proliferation via miR29a-3p. Cells were plated in a 96-well plate and transfected with si-NC, si-H19, miR-29-3p inhibitor, or si-H19 + miR-29-3p inhibitor. CCK-8 assay was used to detect the cell proliferation at the indicated time points. All data were represented as the mean $\pm \mathrm{SE}(\mathrm{n}=3),{ }^{*} \mathrm{p}<0.05$ vs si-NC.
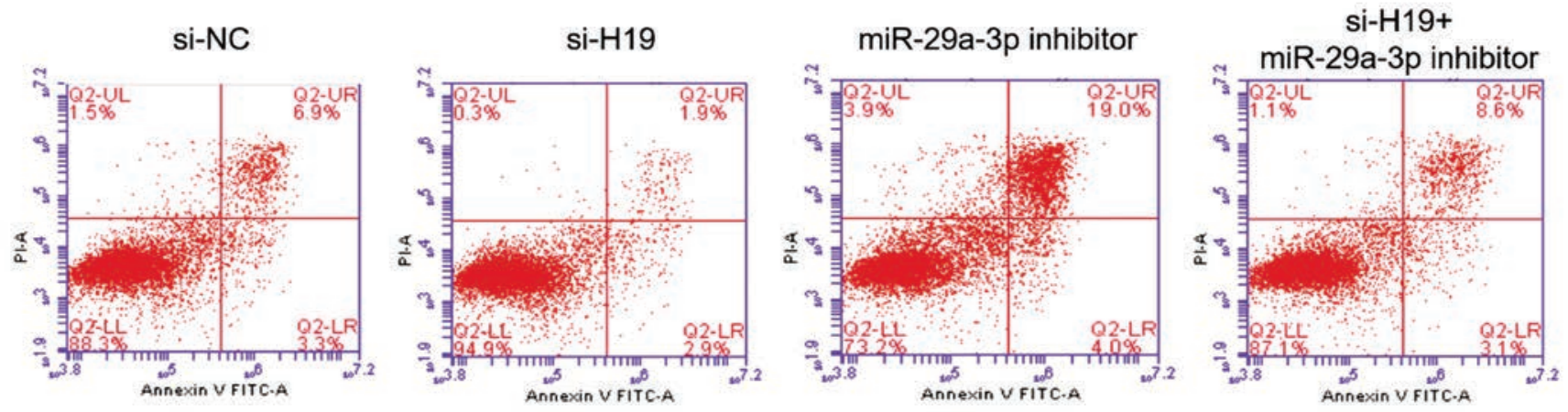

Figure 5. IncRNA H19 regulated the cell apoptosis through miR-29a-3p. Annexin V was used to monitor the in vitro cell apoptosis after transfection with si-NC, si-H19, miR-29-3p inhibitor, or si-H19 + miR-29-3p inhibitor. Osteoclasts cell apoptosis was significantly inhibited after cells were transfected with si-H19. However, co-transfecting with miR-29a-3p inhibitor and siRNA-H19 obligated the effects of siRNA-H19 on the cell proliferation. 
The lncRNA H19/miRNA axis regulatory function has been found to play important roles in different diseases. IncRNA H19/miR-675/PPAR $\alpha$ axis has been indicated to regulate liver cell injury and energy metabolism remodeling induced by hepatitis BX protein via Akt/mTOR signaling. ${ }^{21}$ In addition, lncRNA H19 has been verified to function as an aquaporin 1 competitive endogenous RNA to regulate miR-874 expression in lipopolysaccharide (LPS) sepsis. ${ }^{22}$ In this study, we also investigated the combination function of lncRNA H19/ miR-29a-3p in OP. Our data also indicated that the miR-29a-3p was significantly downregulated in OP plasma compared with that in the health control samples. The miR$29 a-3 p$ inhibitor obligated the effect of si-H19 on pro-inflammatory mediators, cell proliferation and cell apoptosis. However, it is worthy to consider the dysfunction of lncRNA H19/miR-29a-3p axis downstream genes, and the in vivo study in the future study.

To sum up, IncRNA H19/miR-29a-3p axis contributes to the development of OP. Our findings also offered a better understanding of OP development and a potential therapeutic target for OP therapeutic intervention.

\section{Acknowledgements}

The authors express their gratitude to MeiJiaYouKe Biotech Inc. in Wuhan for the proof-reading and grammatical errors correction.

\section{References}

1. Simsek G, Uzun H, Karter Y, Aydin S, Yigit G. Effects of osteoporotic cytokines in ovary-intact and ovariectomised rats with induced hyperthyroidism; is skeletal responsiveness to thyroid hormone altered in estrogen deficiency? Tohoku J Exp Med. 2003;201:81-9.

2. Zhao L, Mao Z, Schneider LS, Brinton RD. Estrogen receptor beta-selective phytoestrogenic formulation prevents physical and neurological changes in a preclinical model of human menopause. Menopause 2011;18:1131-42.

3. Gambacciani M, Levancini M. Hormone replacement therapy and the prevention of postmenopausal osteoporosis. Prz Menopauzalny 2014;13:213-20.

4. Li X, Liu R, Yang J, Sun L, Zhang L, Jiang Z, et al. The role of long noncoding RNA H19 in gender disparity of cholestatic liver injury in multidrug resistance 2 gene knockout mice. Hepatology 2017;66:869-84.

5. Grillone K, Riillo C, Scionti F, Rocca R, Tradigo G, Guzzi PH, et al. Non-coding RNAs in cancer: platforms and strategies for investigating the genomic "dark matter". J Exp Clin Cancer Res 2020;39:117.

6. Sharma U, Barwal TS, Malhotra A, Pant N, Vivek, Dey D, et al. Long non-coding RNA TINCR as potential biomarker and therapeutic target for cancer. Life Sci 2020;257:118035.

7. Zhang L, Xu X, Su X. Noncoding RNAs in cancer immunity: functions, regulatory mechanisms, and clinical application. Mol Cancer 2020;19:48.
8. Yu H, Zhou W, Yan W, Xu Z, Xie Y, Zhang P. LncRNA CASC11 is upregulated in postmenopausal osteoporosis and is correlated with TNF-alpha. Clin Interv Aging 2019;14:1663-9.

9. Han Y, Liu C, Lei M, Sun S, Zheng W, Niu Y, et al. LncRNA TUG1 was upregulated in osteoporosis and regulates the proliferation and apoptosis of osteoclasts. J Orthop Surg Res 2019;14:416.

10. Ghapanchi J, Ranjbar Z, Mokhtari MJ, Koohpeima F, Derakhshan M, Khademi B, et al. The LncRNA H19 rs217727 polymorphism is associated with oral squamous cell carcinoma susceptibility in Iranian population. Biomed Res Int 2020;2020:1634252.

11. Zheng JF, Guo NH, Zi FM, Cheng J. Long noncoding RNA H19 promotes tumorigenesis of multiple myeloma by activating BRD4 signaling by targeting microRNA 152-3p. Mol Cell Biol 2020;40:e00382-19.

12. Wang J, Zhao L, Shang K, Liu F, Che J, Li H, et al. Long noncoding RNA H19, a novel therapeutic target for pancreatic cancer. Mol Med 2020;26:30.

13. Bartel DP. MicroRNAs: target recognition and regulatory functions. Cell 2009;136:215-33.

14. Zhang N, Hu X, He S, Ding W, Wang F, Zhao Y, et al. LncRNA MSC-AS1 promotes osteogenic differentiation and alleviates osteoporosis through sponging microRNA-140-5p to upregulate BMP2. Biochem Biophys Res Commun 2019;519:790-6.

15. He H, Wang N, Yi X, Tang C, Wang D. Long non-coding RNA H19 regulates E2F1 expression by competitively sponging endogenous miR-29a-3p in clear cell renal cell carcinoma. Cell Biosci 2017;7:65.

16. Yang X, Yang J, Lei P, Wen T. LncRNA MALAT1 shuttled by bone marrow-derived mesenchymal stem cells-secreted exosomes alleviates osteoporosis through mediating microRNA34c/SATB2 axis. Aging (Albany NY) 2019;11:8777-91.

17. Fu H, Tan J, Yin Q. Effects of recombinant adeno-associated virus-mediated CD151 gene transfer on the expression of rat vascular endothelial growth factor in ischemic myocardium. Exp Ther Med 2015;9:187-90.

18. Graef JL, Rendina-Ruedy E, Crockett EK, Ouyang P, Wu L, King JB, et al. Osteoclast differentiation is downregulated by select polyphenolic fractions from dried plum via suppression of MAPKs and Nfatc1 in mouse C57BL/6 primary bone marrow cells. Curr Dev Nutr 2017;1:e00406.

19. Wang CG, Liao Z, Xiao H, Liu H, Hu YH, Liao QD, et al. LncRNA KCNQ1OT1 promoted BMP2 expression to regulate osteogenic differentiation by sponging miRNA-214. Exp Mol Pathol 2019;107:77-84.

20. Li X, Wang H, Zhang Y, Zhang J, Qi S, Zhang Y, et al. Overexpression of lncRNA H19 changes basic characteristics and affects immune response of bovine mammary epithelial cells. PeerJ 2019; 7:e6715.

21. Tang J, Yan T, Bao Y, Shen C, Yu C, Zhu X, et al. LncRNA GLCC1 promotes colorectal carcinogenesis and glucose metabolism by stabilizing c-Myc. Nat Commun 2019;10:3499.

22. Fang Y, Hu J, Wang Z, Zong H, Zhang L, Zhang R, et al. LncRNA H19 functions as an Aquaporin 1 competitive endogenous RNA to regulate microRNA-874 expression in LPS sepsis. Biomed Pharmacother 2018;105:1183-91.

Received for publication: 23 June 2020. Accepted for publication: 18 September 2020.

This work is licensed under a Creative Commons Attribution-NonCommercial 4.0 International License (CC BY-NC 4.0).

CCopyright: the Author(s), 2020

Licensee PAGEPress, Italy

European Journal of Histochemistry 2020; 64:3155

doi:10.4081/ejh.2020.3155 